\title{
Die Arbeit ist fertig - was nun?
}

\author{
Empfehlungen zum Umsetzen von Ergebnissen aus \\ Graduierungsarbeiten
}

Promotions-, Master- und auch Bachelorarbeiten enthalten eine Fülle von

Ideen. Aber wie viele davon werden umgesetzt? Sicher besteht für den

Verfasser das unmittelbare Interesse zunächst darin, seine Befähigung für das wissenschaftliche Arbeiten nachzuweisen und diese auch angemessen bestätigt zu bekommen. Bleibt es aber bei diesem Anspruch, so wird ein Großteil der Arbeiten letztlich zum „Ideengrab“. Der Verfasser muss selber erkennen: Die Idee als solche entfaltet noch keine Wirkung. Auf ihre Umsetzung kommt es an. Im vorliegenden Text werden dafür Anregungen vorgestellt.

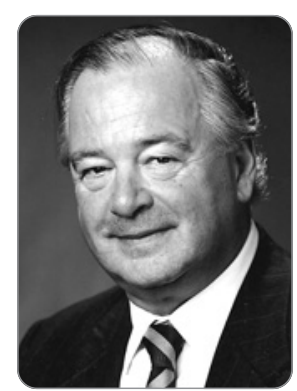

Prof. Dr. Günter Lehmann

ist Vizepräsident des Europäischen Instituts für postgraduale Bildung an der TU Dresden (EIPOS) sowie Dozent an der Dresden International University (DIU). Bevorzugte Forschungsgebiete: Techniken wissenschaftlichen Arbeitens, Ingenieurpädagogik.

\section{Stichwörter: Ansprechpartner, Erfolgsfaktoren, Gruppendiskussion, Moderation, Präsentation, Umsetzungserfolg}

Für einen Weg des Verwertens von Ergebnissen, dem Publizieren, hat der Autor in der WiSt (Nr. 5/2018, S. 54 ff.), einige Vorschläge angeboten. Ein anderer Weg wird hier zum Umsetzen von Ergebnissen in nützliche Lösungen für Unternehmen, Kliniken, Verwaltungen, Vereinen oder Verbänden - im Folgenden als Organisation bezeichnet - beschrieben. Dafür stehen aktuelle Erfahrungen der Verfasser von Promotions- und Masterarbeiten, im Einzelfall auch Bachelorarbeiten, zur Verfügung, die mit ihren umgesetzten Ideen Veränderungen in der jeweiligen Organisation bewirkt haben.

Im Artikel werden wesentliche Erfolgsfaktoren reflektiert, typische Ansprechpartner in der Organisation charakterisiert und deren Resonanzstellen gekennzeichnet. Eine Checkliste soll den Handlungsvollzug unterstützen. Mit der Gruppendiskussion wird ein Instrument für die Umsetzung von Ideen vorgestellt. Abschließend werden Bedingungen für den Umsetzungserfolg in der Organisation genannt.

\section{Erfolgsfaktoren für das Umsetzen}

Bei der Analyse des erfolgreichen Vorgehens zeigen sich eine Reihe von Faktoren für die Ergebnisumsetzung (vgl. Depiereux, 2017; Convey et al., 2017):

- Mut

Am Anfang steht die eigene Überzeugung von der Nützlichkeit der erreichten Ergebnisse, die letztlich in der Disputation der Arbeit ihre Bestätigung durch die Kommission erfahren hat. Das führt zur Entscheidung des Verfassers, die Umsetzung in der Organisation energisch zu betreiben. Empfehlung: Gib deinem Vorschlag eine Chance!

- Agilität Ist die Entscheidung gefallen, ist rasch zu handeln, keine Zeit zu verlieren. Die Chancen auf Umsetzung werden in Zeiten der raschen Veränderung des Bestehenden und der digitalen Transformation mit zunehmender Dauer geringer. Empfehlung: Prüfe zügig die Chancen deines Vorschlags!

- Fokus

Erfahrungsträger warnen davor, die erste Vorstellung der Ergebnisse vor einem größeren, heterogenen Kreis vorzunehmen. Die Präsentation sollte zunächst in kleineren Gruppen von Ansprechpartnern in der Organisation vollzogen werden, die auf spezifische Weise zur Umsetzung beitragen können, z. B. eine Empfehlung für die Annahme des Veränderungsvorschlags geben. 
Empfehlung: Konzentriere dich auf homogene oder auch heterogene Kleingruppen.

- Empathie

Die gezielte Ansprache der jeweiligen Gruppe erfordert die Kenntnis ihrer Resonanzstellen. Davon ausgehend sind jene Aussagen aus der Umsetzungsidee auszuwählen, die für Personen der Gruppe von besonderer Bedeutung sind und deshalb im Mittelpunkt der Präsentation stehen sollten (siehe dazu Abschnitt 4).

Empfehlung: Ermittle die Resonanzstellen der Ansprechpartner!

- Teamwork

Das schrittweise Vorgehen offenbart recht bald, welche Gruppen bzw. Einzelpersonen die Umsetzung unterstützen. Das Verbinden mit den Unterstützern beim weiteren Vorgehen, das Vernetzen innerhalb aber auch außerhalb der Organisation bietet zusätzliche Erfolgschancen.

Empfehlung: Vernetze dich innenhalb und außerhalb der Organisation!

- Zielstrebigkeit

Eine auf Veränderung des Bestehenden gerichtete Umsetzungsidee hat in der Regel stets Hindernisse zu überwinden. Skepsis oder gar Ablehnung bei einigen Ansprechpartnern können zu Rückschlägen führen. Vor allen durch Einstellungen bedingte Vorbehalte können im Verein mit Unterstützern durch geduldiges Überzeugen mit dem Verwenden einstellungskonformer Informationen überwunden werden.
Empfehlung: Gib deinen Vorschlag nicht frühzeitig auf!

- Feedback

Die Gründe für Zustimmung oder Ablehnung sind sorgfältig $\mathrm{zu}$ sammeln, auszuwerten und daraus entsprechende Schlussfolgerungen zu ziehen. Eine Ablehnung soll nicht kommentarlos hingenommen, sondern hinterfragt und für das weitere Vorgehen beachtet werden.

Empfehlung: Verlange eine Einschätzung deines Vorschlags!

\section{Ansprechpartner in der Organisation}

Bei dem Versuch, Ansprechpartner in der Organisation in typischen Gruppen zusammen- zufassen, stehen ihre potenziellen Möglichkeiten für die Unterstützung des Umsetzungsvorschlags im Vordergrund. Das führt zu folgenden fünf Zielgruppen für die Ansprache:

\section{- Veranlasser}

Erkennen, dass zur Lösung bestimmter Probleme in der Organisation externe Hilfe benötigt wird. Von ihnen gehen Anstöße für Veränderungen aus. Sie sind offen für Umsetzungsvorschläge.

\section{- Fachkompetente}

Kennen sich mit dem Umsetzungsvorschlag aus, neigen zur gründlichen fachlichen Prüfung. Von ihnen gehen wesentlich Informationen an die Entscheidungsträger aus.

\begin{tabular}{|c|c|c|}
\hline Ansprechpartner & Unterstützungspotential & Aussagen von besonderer Bedeutung \\
\hline Veranlasser & $\begin{array}{l}\text { Anstoß für Veränderungen } \\
\text { geben }\end{array}$ & $\begin{array}{l}\text { Veränderung ist notwendig, Problem ist klar } \\
\text { gekennzeichnet, Umsetzungsvorschlag setzt an der } \\
\text { Ursache an, ist schlüssig und effektiv. }\end{array}$ \\
\hline $\begin{array}{l}\text { Fach- } \\
\text { kompetenter }\end{array}$ & $\begin{array}{l}\text { Empfehlung an } \\
\text { Entscheider geben }\end{array}$ & $\begin{array}{l}\text { Umsetzungsvorschlag ist fachlich solide, } \\
\text { Wechselwirkung mit anderen Problemen bzw. } \\
\text { Themen ist erkennbar, kritische Details bei der } \\
\text { Umsetzung sind beachtet. }\end{array}$ \\
\hline Beeinflusser & $\begin{array}{l}\text { Auf Entscheider } \\
\text { Einfluss nehmen }\end{array}$ & $\begin{array}{l}\text { Imagegewinn ist erkennbar, Erfolg ist messbar, } \\
\text { Vorschlag ist realisierbar und ökonomisch zu } \\
\text { vertreten, Interessen der Beeinflusser sind } \\
\text { berücksichtigt. }\end{array}$ \\
\hline Entscheider & $\begin{array}{l}\text { Über Umsetzungsvorschlag } \\
\text { entscheiden }\end{array}$ & $\begin{array}{l}\text { Vorschlag passt in die Strategie, } \\
\text { Kosten/Nutzenverhältnis ist } \\
\text { positiv, Umsetzungszeit ist vertretbar, Risiko } \\
\text { beherrschbar (Exit-Strategie), kein Verlust an } \\
\text { Popularität. }\end{array}$ \\
\hline Nutzer & $\begin{array}{l}\text { Den Umsetzungsvorschlag } \\
\text { realisieren }\end{array}$ & $\begin{array}{l}\text { Zustimmung durch die Leitung liegt vor, Inhalt und } \\
\text { Aufwand der Veränderung sind erkennbar, Vorteile } \\
\text { überwiegen, persönliche } \\
\text { Auswirkungen sind erkennbar. }\end{array}$ \\
\hline
\end{tabular}




\section{- Beeinflusser}

Sind als Aktionäre, Gesellschafter, Berater oder Angehörige spezieller Abteilungen nur indirekt von dem Umsetzungsvorschlag betroffen. Sie können aber aufgrund ihrer Sonderstellung Einfluss auf den bzw. die Entscheider ausüben.

\section{- Entscheider}

Treffen die Entscheidung über den Umsetzungsvorschlag, gegebenenfalls unter dem Einfluss der anderen Zielgruppen.

\section{- Nutzer}

Sind von dem Umsetzungsvorschlag direkt betroffen, müssen ihn realisieren, müssen mit ihm arbeiten.

Jede der fünf Zielgruppen hat eine spezielle Interessenlage in Bezug auf den Umsetzungsvorschlag. Sie zu erkunden und gezielt anzusprechen, ermöglicht jene Aussagen aus dem Umsetzungsvorschlag zu bestimmen, die für die Zielgruppe von besonderer Bedeutung sind und deshalb im Mittelpunkt der Präsentation stehen. Dazu werden in Auswertung zahlreicher Gespräche mit Absolventen sowie eigenen Erfahrungen in $A b b .1$ (insbes. Spalte 3) Vorschläge unterbreitet.

Für den Fall, dass die wissenschaftliche Arbeit in der Organisation betreut wird, kann eine besondere Zielgruppe entstehen. Wer hat das Thema befürwortet oder vergeben und wer hat die Arbeit in der Organisation betreut? Das könnte zu einem „Kernteam" führen, mit dem begonnen wird, die Identifikation der Interessen vorzunehmen und über die Umsetzung in der Organisation zu diskutieren (vgl. Bauch, 2018).

\section{Gruppendiskussion}

Natürlich kann der Umsetzungsvorschlag dem Entscheider unter Betonung der Aussagen, die für ihn von Bedeutung sind (vgl. $A b b .1$ ), sofort präsentiert werden. Allerdings muss man ihm zubilligen, dass er dem Vorschlag in der Regel nicht sofort zustimmen kann, sondern im besten Fall eine Prüfung zusagen wird. Diese Prüfung führt er in der Regel nicht selber durch. Er delegiert sie an einen Dritten und schon ist der Umsetzungsvorschlag seinem Schöpfer aus der Hand genommen. Wer ist der Prüfer, ist er vielleicht mit anderen Dingen stark beschäftigt, ist er skeptisch gegenüber Veränderungen oder lehnt er sie gar gänzlich ab? Erfahrungsgemäß wehren sich Menschen nicht gegen die Veränderung, sondern dagegen, verändert zu werden. Widerstände und Ängste gibt es bei nahezu allen Veränderungen. Man denke nur an die Angst vor dem Verlust der Verantwortung, an überforderte Führungskräfte, an die Angst vor Substitution von Mitarbeitern oder vor verstärkter Kontrolle und Transparenz. Die Gefahr ist groß, dass der Vorschlag irgendwo versickert. Deshalb sollte der Autor sein
Projekt in der Hand behalten und die Entscheidungsvorbereitung, also die Prüfung in eigener Sache, selbst organisieren.

Hierbei bewährt sich das Instrument der Gruppendiskussion (vgl. ausführlich dazu bei Loos et al., 2012; Scholl, 2015, May et al., 2018). Die Gruppe, mit der der Verfasser seinen Umsetzungsvorschlag diskutiert, besteht aus Angehörigen der Organisation und kann

- homogen - also beispielsweise 2 bis maximal 3 Sachkompetente oder

- heterogen - zum Beispiel 1 Veranlasser, 1 Sachkompetente und 1 Umsetzer

zusammengesetzt sein.

Mit der fokussierten Gruppendiskussion zur Umsetzung des Veränderungsvorschlags werden (allerdings schrittweise) zwei Ziele verfolgt:

1. Erhebung von Meinungen, Orientierungs- und Bedeutungsmustern zum Umsetzungsvorschlag durch Interaktion der Teilnehmer. Im Ergebnis werden die Chancen der Umsetzung unter den konkreten Bedingungen der Organisation erkannt.

2. Modifizierung des Umsetzungsvorschlags im Ergebnis übereinstimmender Meinungen (Konsens) und Akzentuierung der Präsentation vor dem Entscheider. Im Ergebnis sollen die Chancen für die Umsetzung erhöht werden.

Auf diese Weise kann die Gruppendiskussion durch einen auf dem Konsens verschiedener Gruppenmitglieder beruhenden Vorschlag die Entscheidung über die Umsetzung befördern. Dabei findet die ursprüngliche Idee in der Regel Erweiterung, Ergänzung oder partielle Korrektur durch die Beiträge in der Diskussion. Der Verfasser kann in der Folge nicht mehr die alleinige Urheberschaft für den modifizierten Umsetzungsvorschlag beanspruchen. Will er aber seine ursprüngliche Idee unverfälscht aufbewahren, sichert er das durch Publizieren in der Fachöffentlichkeit.

Bei der Durchführung der Gruppendiskussion hat sich die Moderationsmethode bewährt. In einem strukturierten Verfahren stimuliert und nutzt ein Moderator das Ideenpotential der Gruppenmitglieder in Richtung auf die oben genannten Ziele aus und sichert dabei ein faires und konstruktives Zusammenwirken in der Gruppe (vgl .ausführlich dazu bei Lehmann, 2017, S. 48-70). Das moderierte Gespräch enthält vor allem folgende Schwerpunkte: Vorstellen des Umsetzungsvorschlags, Meinungsaustausch, Meinungsbildung, Ideen zur Modifizierung des Vorschlags, Bewertung der Ideen, Konsensfindung, Dokumentation des modifizierten Vorschlags und die Strategie der Präsentation vor den Entscheidern.

Wer sollte ein solches Gespräch leiten, wer übernimmt die Rolle des Moderators? Alle Erfahrungen verweisen in diesem Falle auf den Schöpfer des Vorschlags selbst. Aller- 


\section{Ziel}

\section{Konsens}

Gibt es Einverständnis?

Zusammenfassung

Konsens

Sind alle dafür?

Zusammenfassung

Konsens

Sind Sie einverstanden?

Zusammenfassung

Spielregeln

\section{Start}

Abb. 2: Konsens feststellen

dings muss er dabei inhaltliche Zurückhaltung üben. Stattdessen setzt er Impulse für die Diskussion und sorgt für eine Grundordnung in der Diskussion.

Auf das Verhalten des Moderators bezogen verläuft eine Gruppendiskussion in vier Phasen:

- Erste Phase:

In der Eröffnung sollte der Moderator nach Schaffen einer einheitlichen Informationsgrundlage für seinen Umsetzungsvorschlag die Gruppe rasch untereinander ins Gespräch bringen. Insbesondere vermeidet er individuelle Befragungen oder Zwiegespräche mit einzelnen Teilnehmern. Seine Eröffnungsfragen richten sich im thematischen Rahmen stets an alle.
- Zweite Phase:

Im Verlaufe der Diskussion hält der Moderator sich weitgehend inhaltlich zurück. Um Missverständnissen vorzubeugen oder ins Detail zu gehen, stellt er Nachfragen und regt systematisches Vorgehen im inhaltlichen und methodischen Bereich an. Die Vereinbarung bestimmter Regeln für die Diskussion, auch beispielsweise der Verzicht auf Kritik während der Ideenproduktion kann dazu führen, dass einzelne Teilnehmer ihre Unzufriedenheit mit dem Arbeitsfortschritt oder der methodischen Führung des Moderators zwar unterdrücken, aber geistig Abschied von der Zusammenarbeit genommen haben. Um dem vorzubeugen sollte der Moderator im Verlaufe der Zusammenarbeit etappenweise (vgl. Abb. 2):

- feststellen, was bis zum jeweiligen Zeitpunkt bereits erreicht wurde (Zusammenfassung) und

- zugleich nachfragen, ob bis hierher Einverständnis mit dem Vorgehen (Konsens) besteht.

Sofern es Kritikpunkte gibt, sind diese vor Fortsetzung der inhaltlichen Arbeit zu klären.

- Dritte Phase:

Sobald in der Meinungsäußerung eine gewisse Erschöpfung, ein Sättigungsgrad im Ideenaustausch spürbar wird, setzt der Moderator Impulse. Besonders geeignet dafür sind provozierende Fragen, das Aufzeigen von Widersprüchen aber auch das Strukturieren der erreichten Ergebnisse mit der Offenbarung von Lücken oder Schwachstellen. Gebräuchlich sind auch instrumentelle Hilfen, um Vollständigkeit zu erzielen - beispielsweise die Empfehlung von Checklisten oder Leitfäden. Im Bedarfsfall regt er auch die Diskussion zu weiteren Aspekten mit Themenbezug an. Dabei kann ein Strukturbild (Mind Map), wie beispielsweise in $A b b .3 \mathrm{zu}$ einem Vorschlag für das Erschließen von Wachstumsressourcen eines Unternehmens helfen, die notwendigen Ergänzungen vorzunehmen.
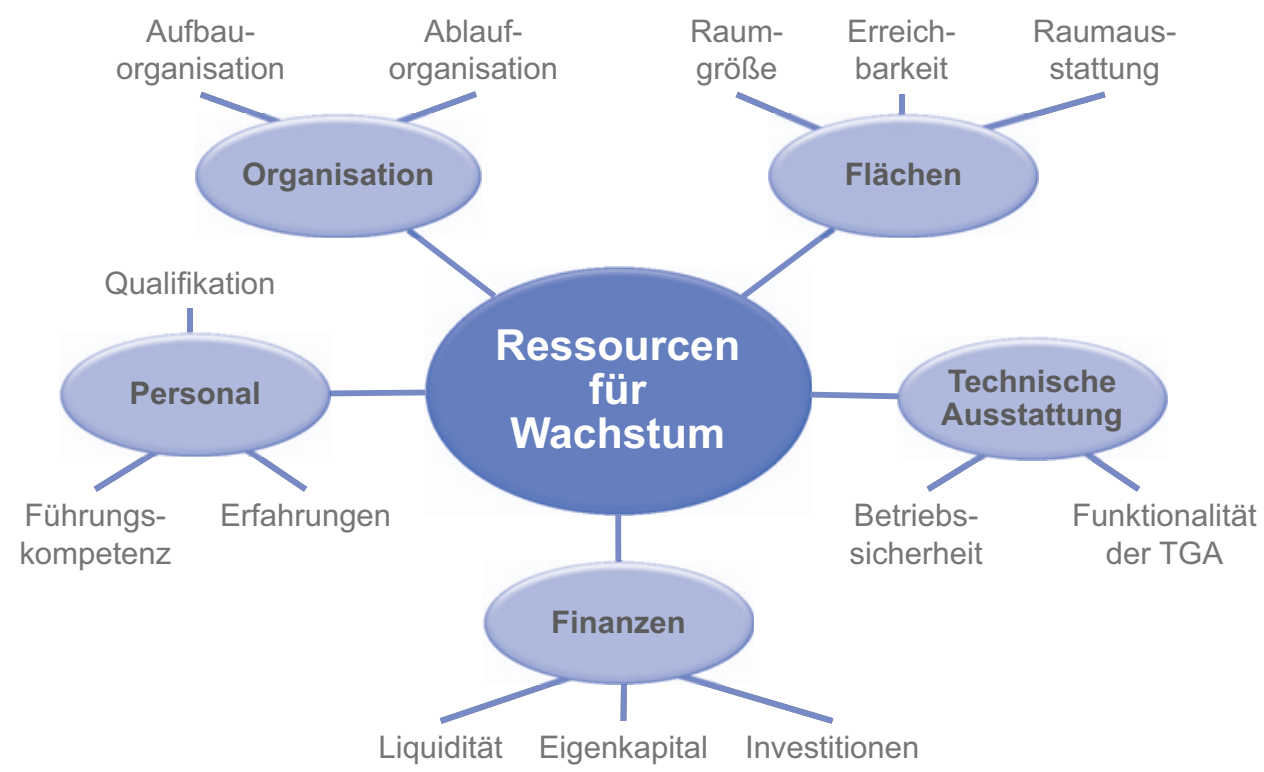

Abb. 3: Strukturbild: Beispiel Wachstumsressourcen für ein Unternehmen 


\section{- Vierte Phase:}

In der letzten Phase ist der Umsetzungsvorschlag ausführlich besprochen. Die Meinungsäußerungen der Gruppenmitglieder werden jetzt gebündelt. Die Modifizierungen (Veränderungen und Ergänzungen) erfolgen weitgehend einvernehmlich, ebenso die Abstimmung über die Argumentationslinien für die Präsentation. Obwohl der Moderator in dieser Phase deutlich direktiver vorgeht vermeidet er es, in der Bewertung der Ergebnisse zu dominieren. Wertvoll sind für ihn auch die Vorschläge der Gruppenmitglieder für seine Präsentation vor dem Entscheider - aber: Was und wie präsentiert wird, das entscheidet er selbst!

Eine gut vorbereitete Gruppendiskussion überschreitet in der Regel nicht die Dauer von drei Stunden. Dabei bewährt es sich, den Gruppenmitgliedern den Umsetzungsvorschlag in kurz gefasster Form vor der Sitzung zuzuschicken, so dass die Eingangsinformation dann kürzer ausfallen kann, jedoch nie eingespart werden sollte. Ein kurzer Fragebogen zu Chancen und Risiken des Umsetzungsvorschlags (max. eine Seite) kann diese Vorabsendung begleiten.

Zwei Empfehlungen schließen die Ausführungen zur moderierten Gruppensitzung ab:

- Erstens sollte der Entscheider nicht als Moderator und möglichst auch nicht als Mitglied der Gruppe nominiert werden (Chefs spalten mitunter die Gruppe in Pro und Kontra). Dagegen ist seine Einflussnahme auf die Zusammensetzung der Gruppe erwünscht.

- Zweitens ist unter allen Umständen die Leitung der Organisation über die Durchführung einer Gruppendiskussion zur Vorbereitung der Leitungsentscheidung in Kenntnis zu setzen, möglichst ihre Zustimmung bzw. Genehmigung zu erlangen.

\section{Präsentation}

Selbstverständlich darf sich der Autor die Präsentation des Umsetzungsvorschlags, auch wenn er im Ergebnis der Gruppenarbeit modifiziert wurde, nicht aus der Hand nehmen lassen. Allerdings ist die Begleitung durch ein Mitglied der Gruppe (möglichst mit Zustimmung des Entscheiders) zu empfehlen.

Die Präsentation vor den Entscheidern folgt anderen Prämissen als die vor der Prüfungskommission. Während es bei der Disputation an der Hochschule vor allem um den Nachweis der wissenschaftlichen Befähigung ging, wird jetzt die überzeugende Darstellung des Nutzens für die Organisation erwartet. Dazu gehören sicher ein internes Standing und das durch die Gruppendiskussion beförderte Selbstvertrauen. Der Umsetzungsvorschlag ist frei von wissenschaftlichen Konventionen, fokussiert auf die Interessen und Ansprüche der anvisierten Zielgruppe (vgl. Abb. 1), neu geordnet, akzentuiert und allgemein verständlich zu präsen- tieren. Auf wissenschaftliche Termini wird weitgehend verzichtet, der wissenschaftliche Diskurs rückt in den Hintergrund. Die Platzierung der Ergebnisse in die Realität der Organisation steht im Vordergrund. Erwartet wird eine Antwort auf die Frage: Welchen Nutzen bringt der Vorschlag für welchen Preis?

Bei der Gestaltung der Präsentation haben sich vor allem folgende Empfehlungen bewährt:

- Im Einstieg wird deutlich markiert, dass eine Veränderung notwendig ist. Der Hauptteil erklärt, was die Veränderung beinhaltet und wie sie mit welchen Konsequenzen erreicht wird. Der Schluss weist das Ergebnis der Veränderung mit erkennbaren Nutzen für die Organisation aus.

- Die zielführende Argumentation folgt weniger einer sachlogischen, sondern eher einer psychologischen Reihenfolge, beispielsweise in den Schritten:

- Konkrete Situation,

- Resultierende Probleme,

- Leistungen des Vorschlags zur Lösung,

- Resultierende Möglichkeiten für innere Leistungsbereiche,

- Perspektive für Außenbeziehungen der Organisation.

- Die Verständlichkeit der vorgetragenen Ergebnisse erfordert

- Konsequentes Orientieren der Aussagen am Nutzen.

- Vereinfachen komplizierter Aussagen durch Überführen in eine bildhafte Form (Visualisierung), durch Modellbildung oder exemplarisches Vorgehen.

- Anschauliche und einfache Sprachgestaltung sowie die Wahl geläufiger Wörter.

\section{Bedingungen für den Umsetzungserfolg}

Auch wenn die Präsentation des Autors zu einer positiven Leitungsentscheidung geführt hat, so ist damit der Veränderungsvorschlag noch lange nicht umgesetzt. Mit einem soliden Change-Management sind nun in Abhängigkeit von der Dimension der vorgeschlagenen Veränderung die dafür notwendigen Bedingungen $\mathrm{zu}$ schaffen. In Anlehnung an Becker, (2012, S. 6 ff.) sind das vor allem die Folgenden:

- Erstens steht die gesamte Leitung der Organisation geschlossen hinter dem Veränderungsvorschlag.

- Zweitens akzeptieren alle Führungskräfte der Organisation den Veränderungsvorschlag und setzen sich glaubwürdig für seine Umsetzung ein.

- Drittens sind alle Betroffenen in der Organisation über den Veränderungsvorschlag zielgruppenspezifisch informiert und zeigen für die erforderlichen Maßnahmen Verständnis.

- Viertens sind die Nutzer des Veränderungsvorschlags im jeweiligen Bereich der Organisation frühzeitig in die Um- 


\section{Emotionale Intelligenz in der Praxis.}

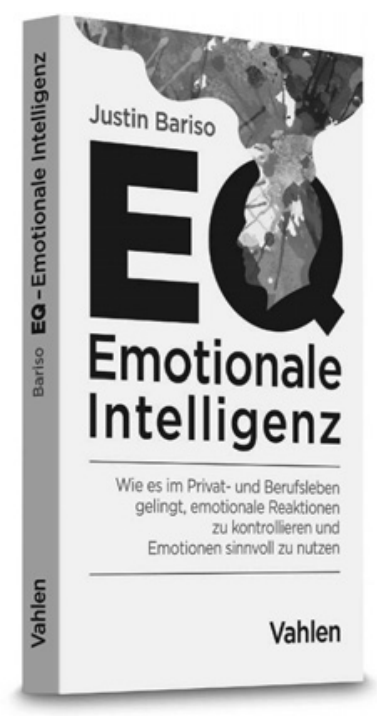

Bariso

EQ - Emotionale Intelligenz

2019. XIV, 183 Seiten. Kartoniert $€ 22,90$

ISBN 978-3-8006-6069-8

\section{In EQ - Emotionale Intelligenz}

veranschaulicht Justin Bariso anhand fundierter Forschungen, moderner Beispiele und persönlicher Geschichten die Kraft des emotionalen Einflusses und wie emotionale Intelligenz in der realen Welt funktioniert - er zeigt darin, wie man Emotionen für sich anstatt gegen sich arbeiten lässt.

\section{Das Buch}

bietet praktische Werkzeuge und Übungen, die zeigen, wie man seine Gefühle kanalisiert, so dass sie uns und unseren (privaten und geschäftlichen) Beziehungen nützlich sind und wie man sich beispielsweise vor Manipulation schützt.

Erhältlich im Buchhandel oder bei:

vahlen.de | Verlag Franz Vahlen $\mathrm{GmbH}$ · 80791 München kundenservice@beck.de | Preise inkl. MwSt. | 170492 setzung eingebunden. Sie sind für die Veränderung sowohl motiviert als auch ausreichend befähigt.

- Fünftens sind alle Stakeholder der Organisation über die Veränderung informiert, um Irritationen und Zielkonflikte $\mathrm{zu}$ vermeiden.

Für den Erfolg ist letztendlich der erkennbare Gestaltungswille des Managements, eine konsequente Umsetzungsorganisation und die intensive Kommunikation mit allen Betroffenen entscheidend. Darauf hat der Verfasser sicher nur begrenzten Einfluss, aber er kann deutlich dazu beitragen, die Veränderungserfolge im Alltag der Organisation sichtbar und bekannt zu machen.

Vielleicht stellt sich jetzt zum Schluss der eine oder andere die Frage: Wie finde ich die Organisation für die Umsetzung meiner Ergebnisse? Für den Absolventen berufsbegleitender Studiengänge ist es in der Regel das aktuelle Einsatzunternehmen, für den Absolventen des Präsenzstudiums der neue Arbeitgeber. In allen anderen Fällen haben schon zwei Tipps von Erfahrungsträgern geholfen:

- Bitte den Betreuer oder Mitglieder der Prüfungskommission um entsprechende Empfehlungen nach der Bestätigung der Ergebnisse in der Disputation.

- Besuche Fachtagungen im Themenbereich deiner Arbeit und spreche gezielt Teilnehmer als Vertreter von Organisationen zu deinem Anliegen an.

Mit bestätigten Ergebnissen im Gepäck und dem festen Willen zur Umsetzung eröffnen sich für den Verfasser auf diesen Wegen oft neue Karrierechancen.

\section{Literatur}

Bauch, U., Umsetzung in der Organisation. Gesprächsprotokoll, Gespräch mit Prof. Dr. Ullrich Bauch, KAISER BAUCONTROL, am 09.08.2018 in Dresden.

Becker, R.., Veränderungen erfolgreich umsetzen - Change-Management in der Wissenschaftsorganisation. Tage des Wissenschaftsmanagements, Vortrag in der Villa Vigoni. In: www.wissenschaftsmanagement-online.de /sites/www.wisse... (Zugriff:. 15.06.2018).

Depiereux, P.: 2018. Von der Idee zur Umsetzung. www.etventure.de/blog /von- der-Idee-zur-Umsetzung7-tip. (Zugriff: 12.07.2018).

Lehmann, G., Lehren mit Erfolg. Ein Praxisratgeber für Dozenten in der beruflichen Weiterbildung, 2., neu bearb. Aufl., Renningen 2017.

Lehmann, G.: 2018. Publizieren - aber wie? Standpunkte und Impulse für das Veröffentlichen wissenschaftlicher Ergebnisse. In: WiSt, Zeitschrift für Wissenschaft und Forschung, Universität Marburg (47) H. 05/2018, S. 54 bis 58. München.

Loos, P.; Schäffer, B.: 2012. Das Gruppendiskussionsverfahren. Theoretische Grundlagen und empirische Anwendung. 2. Auflage, Wiesbaden.

May, G.; Vock, R.; Ruppel, S.: 2018. Gruppendiskussion. www.studi-lektor. de/tipps/qualitative Forschung/Gruppendiskussion (Abruf: 16.07.2018). Scholl, A.: 2015. Die Befragung. 3. Auflage. Konstanz und München.

\section{Vahlen}

